\title{
Estilos y estrategia de enseñanza-aprendizaje de estudiantes universitarios de la Ciencia del Suelo
}

\section{Learning Teaching Styles and Strategies of University Students of Soil Science}

\author{
Leticia Salomé Jiménez Álvarez (*) Isjimenez@utpl.edu.ec \\ Nataly Vega $\left(^{*}\right)$ nlvega@utpl.edu.ec \\ Edwin Daniel Capa Mora $\left(^{*}\right)$ edcapa@utpl.edu.ec \\ Natacha del Cisne Fierro Jaramillo (*) ndfierro@utpl.edu.ec \\ Pablo Quichimbo Miguitama (*) pablo.quichimbo@ucuenca.edu.ec \\ (*) Universidad Técnica Particular de Loja \\ (Recibido: 18 de abril de 2017; Aceptado para su publicación: 23 de julio de 2017)
}

Cómo citar: Jiménez, L. S., Vega, N., Capa, E. D., Fierro, N. del C. y Quichimbo, P. (2019). Estilos y estrategia de enseñanza-aprendizaje de estudiantes universitarios de la Ciencia del Suelo. Revista Electrónica de Investigación Educativa, 21, e04, 1-10. doi:10.24320/redie.2019.21.e04.1935

\section{Resumen}

El propósito de esta investigación fue determinar los estilos de aprendizaje por género y carrera, así como las estrategias de enseñanza más efectivas en educación superior en Ecuador. Se tomó como grupo focal a estudiantes de Edafología de la Universidad Técnica Particular de Loja (UTPL) y se desarrolló un estudio descriptivo, utilizando el Cuestionario Honey-Alonso de Estilos de Aprendizaje (CHAEA); además, se consultaron las estrategias que más impactaron en la enseñanza. Las preferencias fueron de moderadas a bajas para los cuatro estilos de aprendizaje según la carrera y género; las estrategias más efectivas fueron: video, clase magistral y trabajo de campo-laboratorio-aula. Se evidenció que los educandos no aprenden de una única manera, sino que tienen diversos estilos de aprendizaje, por lo que se deberían aplicar estrategias que amplíen el rango de capacidades de aprendizaje.

Palabras clave: Educación superior, aprendizaje, método de aprendizaje, edafología, Ciencias del Suelo.

\section{Abstract}

This study sought to determine learning styles by sex and degree, as well as the most effective teaching strategies in higher education in Ecuador. With a focus group made up of edaphology students from the Private Technical University of Loja (UTPL), a descriptive study was conducted using the Honey-Alonso Learning Styles Questionnaire (CHAEA). The research also explored which teaching strategies had the greatest impact. Preferences by degree and sex were moderate to low for the four learning styles, and the most effective strategies were videos, lectures, and field-laboratory-classroom work. It was shown that students do not learn in just one way, but have different learning styles, and therefore strategies should be applied that respond to a wider range of learning capacities. 


\section{Introducción}

Los suelos desarrollan funciones ecosistémicas claves en el ambiente; es, además, un insumo clave para la producción de alimentos, fibras y biocombustibles, posibilitando así la vida del ser humano y de otros seres (Balks y Zabowski, 2016; Mol y Keesstra, 2012). Como muestra de su importancia han existido declaraciones como la celebración del "Año internacional del suelo" en 2015, además de la declaración de la Década Internacional de los Suelos: 2015-2024 (Organización de las Naciones Unidas para la Alimentación y la Agricultura [FAO, por sus siglas en inglés], 2015; International Union of Soil Sciences [IUSS], 2016). Sin embargo, para garantizar la gestión sostenible de este recurso es vital transmitir e impartir a las próximas generaciones de científicos relacionados con la Ciencia del Suelo los conocimientos y herramientas de vanguardia (Diochon et al., 2017). Gran parte de esta tarea se debería cumplir en las instituciones de educación superior; en el contexto ecuatoriano la ciencia del suelo se imparte en diferentes carreras universitarias, relacionadas con las ciencias biológicas, agropecuarias y ambientales. La importancia de su enseñanza no sólo a nivel nacional sino internacional radica en los múltiples bienes y servicios que el suelo presta a la sociedad y al ambiente (FAO, 2015; Hartemink, 2015). El docente es el responsable de enviar este mensaje a sus alumnos, pero primero debe motivarlos en la sala de clase (Hartemink et al., 2014).

El profesor de la Ciencia del Suelo que domina el conocimiento científico de la materia por su formación académica y experiencia, es parte de la garantía para el éxito del curso, sin embargo que el alumno tenga éxito en su clase no depende sólo de las capacidades del profesor (Wrenn y Wrenn, 2009). Amador y Görres (2004) sostienen que la enseñanza de la Ciencia del Suelo es tradicionalmente una combinación de teoría y práctica, aspecto a incluir cuando se realiza el plan curricular para la enseñanza de esta ciencia. Sin embargo, existen otros aspectos que no se han considerado dentro del proceso de aprendizajeenseñanza, entre ellos las características de los estudiantes que participan en la educación de esta rama, entre las que destacan los estilos de aprendizaje (Quichimbo, Chérrez y Jiménez, 2016).

Los estilos pueden ser entendidos como las diferentes formas de aprender, ver, escuchar, pensar y razonar (Gaona-Flores, Campos-Navarro, Alcalá y Ovalle-Luna, 2014 Pashler, McDaniel, Rohrer y Bjork, 2009) y permiten ver ciertos rasgos o características individuales, pero también permiten agrupar a los estudiantes con características o estilos similares (Hawk y Shah, 2007). Al respecto, es reconocido que los estilos de aprendizaje pueden cambiar dependiendo de factores como la edad, género, carrera, cultura, idioma y otras circunstancias (Joy y Kolb, 2009; Gaona-Flores et al., 2014; García, Sánchez, Jiménez y Gutiérrez, 2012; Maldonado, 2014; Mumford y Honey, 1992; Villalba, 2015). Con esta información los docentes pueden plantear una variedad de actividades que apoyen al aprendizaje (Hawk y Shah, 2007).

Una de las herramientas para el estudio de tales estilos -y que se ha utilizado en el mundo hispanohablante- es el del cuestionario de Honey y Mumford, conocido como Cuestionario Honey-Alonso de Estilos de Aprendizaje (CHAEA) (Alonso, Gallego y Honey, 1995), que se caracteriza por el análisis de actividades de trabajo real y conduce directamente a la identificación de los tipos de comportamiento que reducen la capacidad de un alumno como aprendiz. El cuestionario fue desarrollado tomando como base la teoría de Kolb (Honey y Mumford, 1986), bajo la reflexión de que cada persona origina diversas respuestas y conductas ante el aprendizaje dependiendo de su estilo (Mumford y Honey, 1992).

Se identifican cuatro estilos de aprendizaje según el modelo de Kolb:

El estilo activo lo presentan los individuos generadores de ideas, que tratan nuevos enfoques y buscan nuevas experiencias, son entusiastas y actúan primero sin analizar las consecuencias; en la clasificación de Kolb, este estilo corresponde al de "experiencia concreta"; ellos prefieren estrategias en el aula, como lluvias de ideas, foros de discusión, preguntas con múltiples respuestas, seguir guías y protocolos (García, et al., 2012; Gaona-Flores et al., 2014).

Los reflexivos piensan antes de actuar, son receptivos y analíticos, según Kolb los individuos con este estilo están vinculados a la "observación reflexiva", a ellos les gusta el aprendizaje basado en problemas, foros de discusión, preguntas de múltiples respuestas (Lesmes-Anel, Robinson y Moody, 2001; García, et 
al., 2012; Gaona-Flores et al., 2014).

Los teóricos prueban asunciones básicas, son lógicos, tienden a ser perfeccionistas, sacan conclusiones de la experiencia; en los estilos de aprendizaje de Kolb están asociados a la "conceptualización abstracta", se divierten con preguntas definitivas y con dirección clara de su profesor (García et al., 2012; Wilkinson, Boohan y Stevenson, 2014).

Por último, los pragmáticos aprenden con la práctica, tratan nuevas ideas, son impacientes, planean el próximo paso, se asocian a la "experimentación activa"; en los estilos que propone Kolb, prefieren la exposición en temas, el manejo de paquetes estadísticos, las actividades autodirigidas (Lesmes-Anel et al., 2001; Wilkinson et al., 2014).

Tanto los activos como los reflexivos, teóricos y pragmáticos aprenden también con juegos de roles, métodos de proyectos, métodos de casos, uso de blogs, wikis, google docs y elaboración de mapas conceptuales (García et al., 2012).

Mumford y Honey (1992) indican que los activos y pragmáticos se orientan mejor "haciendo" y los reflexivos y teóricos "pensando"; esto lleva a considerar que los estudiantes tendrán diversas preferencias de aprendizaje como videos, discusiones, clase magistral, lecturas de imágenes visuales y juegos en el aula, entre otros (Hawk y Shah, 2007; Czepula et al., 2016). Conocer los estilos de aprendizaje no es la solución completa para el éxito de los estudiantes (Fazarro, Pannkuk, Pavelock y Hubbard, 2009), pero sí permitirá que el docente aplique diversos recursos educativos que contribuyan a su aprendizaje, porque cada estilo de aprendizaje tiene sus propias fortalezas y debilidades, las que permiten que los estudiantes aprendan de diversas maneras (Dunn y Dunn, 1989).

Por otro lado, los estudiantes aprenden con más efectividad cuando se les enseña utilizando estrategias según sus estilos de aprendizaje predominantes. En un número importante de artículos se menciona el diagnóstico de los estilos de aprendizaje, pero en un número menor se hace referencia a las estrategias de enseñanza (Gallego, 2013). Este estudio examina por primera vez los estilos y las estrategias de aprendizaje de los estudiantes de la Ciencia del Suelo en la Universidad Técnica Particular de Loja (UTPL), como iniciativa para fomentar la innovación en la enseñanza-aprendizaje de esta ciencia.

\section{Método}

El estudio se realizó en la UTPL, situada en la provincia de Loja, al sur de Ecuador. La encuesta se aplicó a los estudiantes matriculados en las asignaturas de Edafología y Conservación de Suelos de las carreras de Ingeniería Agropecuaria (37 estudiantes), de los períodos abril-agosto 2016 y octubre-febrero 2017; y de la carrera de Biología (28 estudiantes), del período octubre 2016-febrero 2017, en el caso de Biología no se aplicó debido a que la oferta del componente es anual. Los estudiantes contestaron de manera voluntaria el cuestionario CHAEA, el cual consta de 80 preguntas dicotómicas cortas que contemplan a los cuatro estilos y están distribuidas de manera aleatoria. El rango de valoración más alto es 20 y la nota alcanzada indica el tipo de estilo; sin embargo, la valoración de las preferencias para los estilos puede ser calificada de acuerdo a las siguientes cinco categorías: muy bajo, bajo, moderado, alto y muy alto (Alonso, et al., 1995) (ver tabla I). Pero la asignación a cada categoría no es lineal y es propia de cada estilo, por lo tanto, los autores del cuestionario han planteado una escala (baremo) propia de calificación y es la que se ha usado para este estudio; adicionalmente se incluyó en el cuestionario información referente a género, edad, carrera y ciclo académico. 
Tabla I. Baremo general abreviado, estilos de aprendizaje

\begin{tabular}{lccccc}
\hline & $\begin{array}{c}10 \% \\
\text { Muy baja }\end{array}$ & $\begin{array}{c}20 \% \\
\text { Baja }\end{array}$ & $\begin{array}{c}40 \% \\
\text { Moderada }\end{array}$ & $\begin{array}{c}20 \% \\
\text { Alta }\end{array}$ & $\begin{array}{c}10 \% \\
\text { Muy alta }\end{array}$ \\
\hline Activo & $0-6$ & $7-8$ & $\begin{array}{c}9-12 \\
\text { Media* }(10.70)\end{array}$ & $13-14$ & $15-20$ \\
Reflexivo & $0-10$ & $11-13$ & $\begin{array}{c}14-17 \\
\text { Media* }(15.37)\end{array}$ & $18-19$ & 20 \\
Teórico & $0-6$ & $7-9$ & $\begin{array}{c}10-13 \\
\text { Media* }(11.3) \\
11-13\end{array}$ \\
Pragmático & $0-8$ & $9-10$ & $\begin{array}{c}14-15 \\
\text { Media*(12.1) }\end{array}$ & $16-20$ \\
\hline
\end{tabular}

*Media general (Alonso et al., 1995).

Las estrategias de enseñanza utilizadas para impartir las asignaturas se describen en la tabla II. Para la selección de las estrategias a aplicar se consideraron básicamente dos aspectos: 1) recurrir a estrategias utilizadas anteriormente en la clase de Edafología y que se considera tuvieron acogida por parte de los estudiantes; 2) el uso de estrategias propuestas por Ferreiro (2014) y Ferreiro y Espino (2014).

Al finalizar el semestre se consultó al grupo de estudiantes sobre cuáles estrategias de enseñanza utilizadas durante el semestre fueron las más efectivas, el 1 significó la mayor preferencia y 13 la menor. Además, se conversó con algunos de ellos para indagar el porqué de la selección de ciertas estrategias.

Tabla II. Estrategias de enseñanzas consultadas

\begin{tabular}{|c|c|}
\hline Estrategia & Definición \\
\hline Videos & $\begin{array}{l}\text { Incluye videos cortos realizados por los estudiantes en clases sobre una temática } \\
\text { específica y videos realizados por el docente que se encuentran en la plataforma. }\end{array}$ \\
\hline Clase magistral & $\begin{array}{l}\text { Es la explicación teórica del docente en cada clase, para ello se utilizaron las } \\
\text { presentaciones en PowerPoint. }\end{array}$ \\
\hline $\begin{array}{l}\text { Trabajo de campo- } \\
\text { laboratorio-aula }\end{array}$ & $\begin{array}{l}\text { Esta estrategia se aplicó durante todo el ciclo, constituye la parte práctica con salidas de } \\
\text { campo, análisis de laboratorio y la sistematización de los resultados para su discusión y } \\
\text { exposición final. }\end{array}$ \\
\hline $\begin{array}{l}\text { Presentaciones } \\
\text { PowerPoint }\end{array}$ & $\begin{array}{l}\text { Es el material utilizado en cada clase, en donde se incluyen imágenes visuales, datos } \\
\text { científicos, diagramas, entre otros. }\end{array}$ \\
\hline $\begin{array}{l}\text { Preguntas de } \\
\text { razonamiento }\end{array}$ & $\begin{array}{l}\text { Son preguntas específicas de reflexión, en donde un estudiante contesta sobre un caso } \\
\text { planteado. }\end{array}$ \\
\hline Infografía & $\begin{array}{l}\text { Es la representación visual que los estudiantes realizan en grupos, utilizando herramientas } \\
\text { informáticas, la infografía fue presentada y explicada al final del ciclo. }\end{array}$ \\
\hline Cuestionarios & $\begin{array}{l}\text { En cada bimestre se pusieron } 2 \text { cuestionarios ( } 4 \text { en total) con varias preguntas para que } \\
\text { los estudiantes las contesten en el entorno virtual de aprendizaje (plataforma). }\end{array}$ \\
\hline $\begin{array}{l}\text { Competencia } \\
\text { entre grupos }\end{array}$ & $\begin{array}{l}\text { Se planteaban trabajos grupales en donde al final se hacían competencias entre grupos y } \\
\text { estudiantes y docente indicaban qué grupo fue mejor y por qué. }\end{array}$ \\
\hline $\begin{array}{l}\text { Participación } \\
\text { individual }\end{array}$ & Son las sugerencias, aportes, preguntas del docente al estudiante o viceversa. \\
\hline Participación grupal & Son actividades de clase y extraclase que los estudiantes desarrollan en grupo. \\
\hline Exposiciones & Se realizaron por grupos con una temática específica, en tiempos definidos. \\
\hline $\begin{array}{l}\text { Información Entorno } \\
\text { virtual de aprendizaje }\end{array}$ & $\begin{array}{l}\text { Es toda la información que se subió a la plataforma: plan docente, información sobre } \\
\text { prácticas de campo, laboratorio, artículos, presentaciones, información en general. }\end{array}$ \\
\hline $\begin{array}{l}\text { Actividades } \\
\text { extraclase }\end{array}$ & $\begin{array}{l}\text { Son actividades que se subieron a través del Eva con tiempos definidos, para los primeros } \\
\text { estudiantes que las realizaron correctamente tuvieron aportes adicionales en su nota } \\
\text { final, las actividades fueron: análisis de datos, juegos, memes, todos relacionados a las } \\
\text { temáticas del curso. }\end{array}$ \\
\hline
\end{tabular}

Fuente: Adaptado de Ferreiro (2014) y Ferreiro y Espino (2014).

Se hizo un análisis descriptivo de los datos para determinar los estilos de aprendizaje por género, carrera y por los cinco niveles de preferencia, considerando el baremo para interpretar las preferencias de estilos de aprendizaje según Honey y Mumford (1986) y Alonso et al. (1995). Para definir las estrategias más efectivas se consideraron las actividades que los estudiantes seleccionaron en los tres primeros lugares 
de las trece opciones que tuvieron.

\section{Resultados y discusión}

De los 65 estudiantes de las carreras de Biología e Ingeniería Agropecuaria, el 95\% completó el cuestionario sobre los estilos de aprendizaje. Del total de participantes $40 \%$ fueron mujeres y $60 \%$ hombres.

En el análisis por género no se observaron diferencias, las preferencias fueron moderadas en los estilos activo y teórico y bajo en los estilos reflexivo y pragmático ( ver figura 1); resultados parecidos obtuvieron Esguerra y Guerrero (2010), quienes encontraron que el género no está relacionado con los estilos de aprendizaje, esto puede darse porque existen otras variables que influyen, como la convivencia, procedencia, el estilo de enseñanza del profesor, la materia, las circunstancias, los compañeros y los padres (Esguerra y Guerrero, 2010; Hussain y Ayub, 2012; Mumford y Honey, 1992).

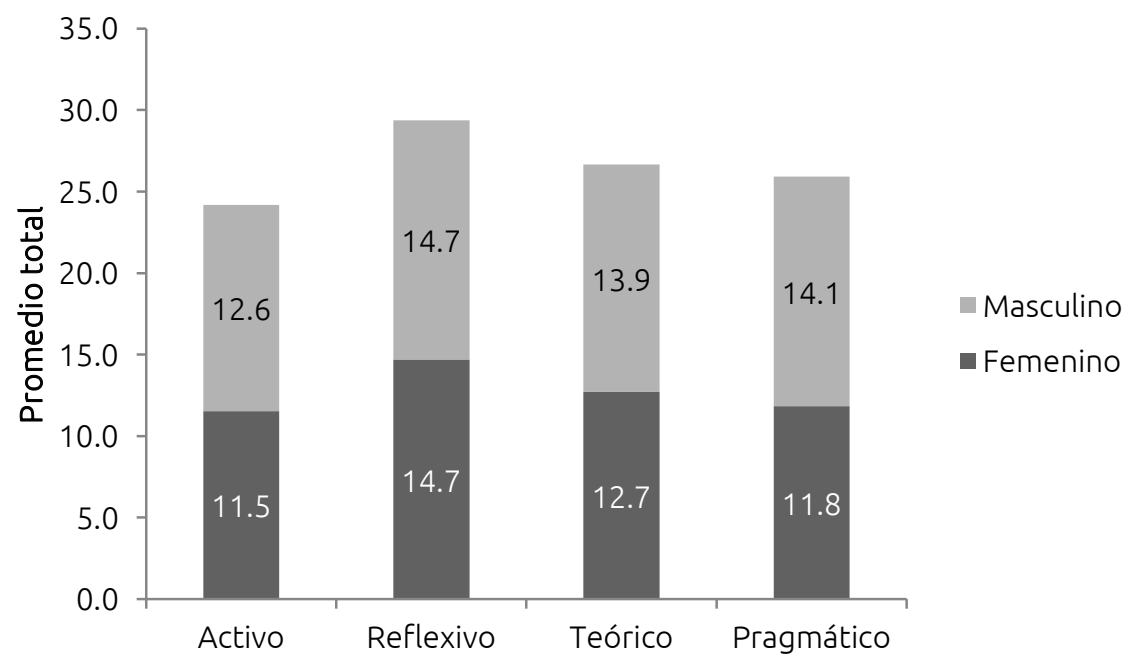

Figura 1. Promedio total de los estilos de aprendizaje por género de los estudiantes de Ingeniería Agropecuaria y Biología

Los participantes pertenecen a las carreras de Biología (42\%) e Ingeniería Agropecuaria (58\%), con 21 años edad promedio. La carrera con el mayor porcentaje fue la de Ingeniería Agropecuaria (75\% hombres y $25 \%$ mujeres), mientras que Biología registró una tendencia distinta: $61 \%$ mujeres y 39\% hombres. Villalba (2015) observó en estudiantes de Biología y licenciatura en Biodiversidad preferencias de "muy baja" en los estilos reflexivo y pragmático y de "moderado" a "bajo" de los estilos activo y teórico, a diferencia del análisis de los estilos de aprendizaje realizado en este estudio por carrera formativa; en la figura 2 se observa que tanto para Agropecuaria como para Biología los estudiantes tienen preferencia moderada por los estilos activo, teórico, pragmático y preferencia baja por el reflexivo (Alonso et al., 1995); así, vemos que es común que los estudiantes muestren una combinación de varios estilos de aprendizaje, lo que nos ayuda a conocer patrones comunes de conducta y amplía la posibilidad de aprender en diversas situaciones (Czepula et al., 2016). En cambio, en un estudio realizado en Ecuador a los estudiantes de la Ciencia del Suelo en la Universidad de Cuenca por Quichimbo, Chérrez y Jiménez (2016) encontraron que la mayoría de los estudiantes son pragmáticos seguido de los activos, ellos comentan que esto se debe a la aplicación práctica de esta ciencia en carreras como Ingeniería Agronómica. 


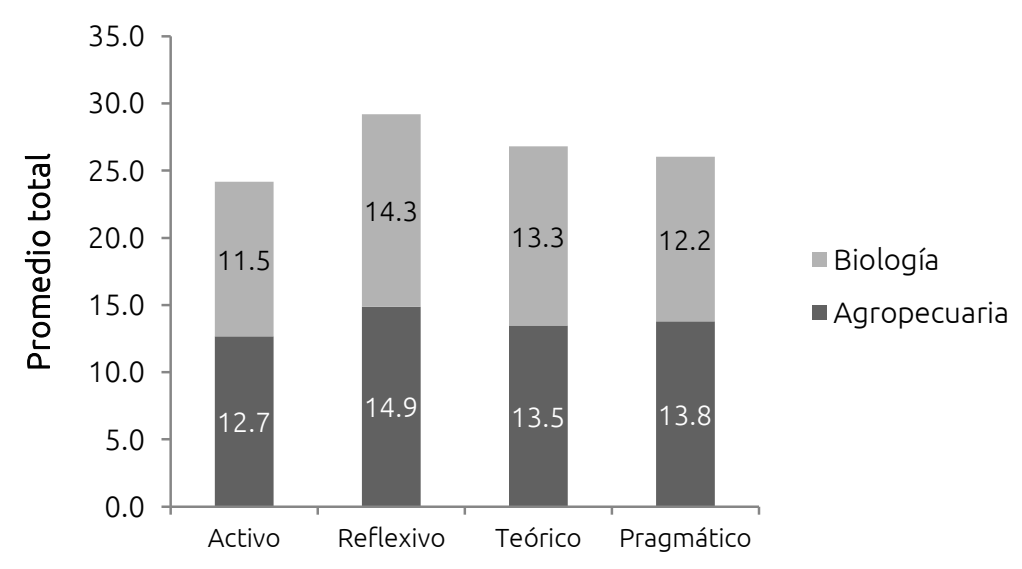

Figura 2. Promedio total de los estilos de aprendizaje de los estudiantes por carrera universitaria (Biología e Ingeniería Agropecuaria)

La preferencia en estilos de aprendizaje no cambió por carrera, estos resultados no coinciden con el estudio realizado por Chiang, Díaz y Arriagada (2016), en donde encontraron que los estudiantes del área de educación tuvieron los estilos en el orden siguiente: activo > pragmático > teórico > reflexivo, en el mismo estudio el orden para los estudiantes de Ciencias fue: teórico > pragmático > activo > reflexivo.

En la figura 3 se visualiza una moderada preferencia de los cuatro estilos de aprendizaje: 50\% para el estilo activo, $48.4 \%$ para el estilo teórico, $41.9 \%$ para el estilo pragmático y $40.3 \%$ para el reflexivo. En todos los casos existieron estudiantes con preferencias "muy alto, alto, moderado, bajo y muy bajo" en los cuatro estilos de aprendizaje, a excepción del teórico con preferencia "muy bajo", donde no existió ningún estudiante.

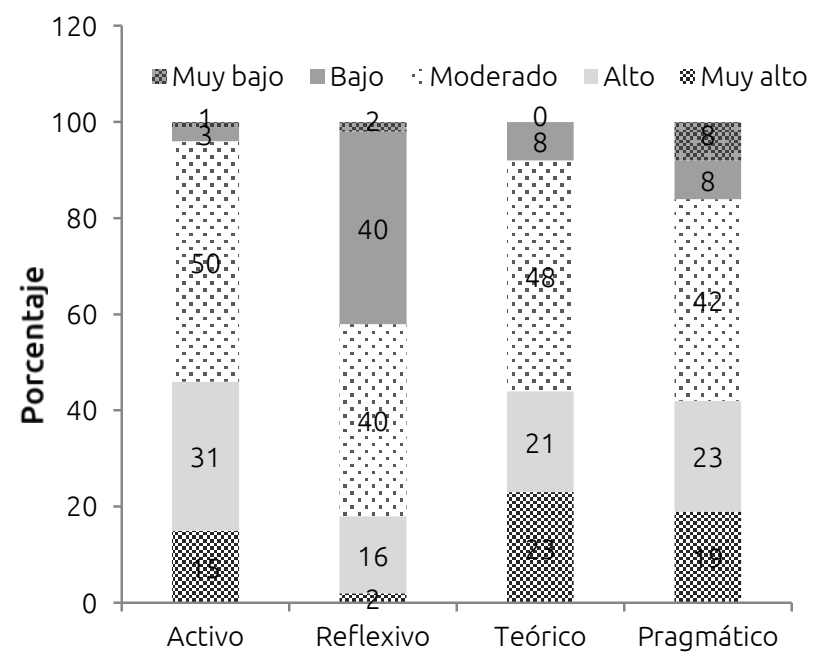

Figura 3. Distribución en porcentaje de estilos de aprendizaje de estudiantes de la Ciencia del Suelo

Un estudio realizado a estudiantes australianos de la Ciencia del Suelo (Field et al., 2011) muestra que las actividades de campo y laboratorio son los escenarios más efectivos para el aprendizaje; en esta investigación el trabajo de campo-laboratorio-aula tuvo aproximadamente el 17\%, valores superiores presentaron los videos y la clase magistral, con cerca del $41 \%$ entre las dos actividades (ver figura 3). 
Hartemink et al. (2014) señalan que la Ciencia del Suelo inicia con la clase magistral y concluye en el campo, en donde se debe crear el interés y hacer más divertida la enseñanza. Estos resultados señalan que la teoría va de la mano de la práctica, siendo las dos actividades trascendentes para el aprendizaje, debido a que una buena base sólida de la teoría contribuirá al éxito de la práctica (Wrenn y Wrenn, 2009). Además, las estrategias empleadas deben repetirse y practicarse en diferentes contextos debido a que el estudio del suelo presenta cierta complejidad porque es una ciencia transversal para otras disciplinas (Field et al., 2011). Existen diversas formas para enseñar la Ciencia del Suelo, Amador y Görres (2004) proponen el aprendizaje basado en problemas, que permite al estudiante mejorar su escritura, debatir y adquirir habilidades resolviendo problemas.

Tabla III. Actividades más efectivas para los estudiantes de Ciencia del Suelo

\begin{tabular}{lc}
\hline Estrategia & $\begin{array}{c}\text { Porcentaje de } \\
\text { respuestas }\end{array}$ \\
\hline Videos & $23.08^{*}$ \\
\hline Clase magistral & $20.00^{*}$ \\
Trabajo campo-laboratorio-aula & $16.92^{*}$ \\
\hline Presentaciones power point & 13.85 \\
Preguntas de razonamiento & 6.15 \\
Infografía & 4.62 \\
Cuestionarios & 3.08 \\
Competencias entre grupos & 3.08 \\
Participación individual & 3.08 \\
Participación grupal & 1.53 \\
Exposiciones & 3.08 \\
Información plataforma (EVA) & 1.53 \\
Actividades extraclase & 0 \\
\hline
\end{tabular}

*Respuestas seleccionadas como opciones 1,2 y 3.

También existieron opiniones divididas y con menores porcentajes, como los cuestionarios, competencias entre grupos, participación individual y exposiciones. Algo notable es el caso del trabajo grupal, una estrategia de aprendizaje que mostró los porcentajes más bajos; con relación a esto, los estudiantes argumentaron su poca participación debido a cruces de horarios o desinterés de ciertos alumnos, incluso en algunos casos solicitaron realizar los trabajos de manera individual; en cambio, en el trabajo realizado por Lesmes-Anel et al. (2001) se evidencia que el trabajo en pequeños grupos fue bastante efectivo.

Las actividades extraclase no fueron consideradas como actividades prioritarias si no como un refuerzo de lo estudiado en clases; un porcentaje similar obtuvo la información que se subió a la plataforma, a pesar de que la tecnología es una herramienta de apoyo para los estudiantes, Wade, Rasmussen y Fox-turnbull (2013) concluyeron en su estudio que la tecnología fue una de las herramientas menos efectivas, quizá porque es un instrumento utilizado en todas las asignaturas de la universidad y gran parte de la explicación se brinda en clases - dos estudiantes mencionaron que no ingresaron nunca a la plataforma, y se pudo corroborar en la plataforma que otros estudiantes ingresaron sólo cada dos o tres semanas. Otras estrategias que también resultaron poco efectivas en la clase de Biología fueron las mencionadas por Acosta y García (2012): lluvia de ideas, mapas y redes conceptuales preguntas dirigidas, organizadores gráficos y preguntas intercaladas.

Como se ha mencionado, existe una interesante gama de estrategias que se pueden utilizar en el aula incluso para los estudiantes de pregrado, como lo es escribir un artículo científico por grupos o de manera individual sobre un proyecto desarrollado en clase (Fox, Kuster y Fox, 2017); sin embargo, sería conveniente seleccionar las que dan más resultados de acuerdo a los estilos de aprendizaje de los estudiantes del curso y no quedarse sólo en la aplicación de un gran número de estrategias de poca efectividad.

Por otra parte, en el análisis de aprobación y reprobación de la asignatura de Edafología el 17.74\% de los estudiantes tuvo calificaciones menores a 28 puntos -lo que equivale a la reprobación de la asignatura. 
Del total de estudiantes, el $81.81 \%$ se ubicó como reflexivo bajo, y de ese porcentaje el $60 \%$ seleccionó los videos como una de las estrategias más exitosas. En cambio, de los estudiantes que obtuvieron más de 34 puntos el 62.5\% fue teóricos alto y muy alto, y pragmáticos muy alto; seguido de moderado a muy alto del estilo activo, lo que de cierta manera explica que los estudiantes con estilos de aprendizaje múltiples suelen obtener mejor puntaje que aquellos en los que predomina un solo estilo de aprendizaje. El 75\% de ellos seleccionó las presentaciones en PowerPoint entre las actividades prioritarias y las actividades extraclase como las menos efectivas. En los estudiantes cuyas notas estuvieron entre 29 y 33 puntos no predominó un estilo de aprendizaje, y entre las actividades más efectivas estuvieron las presentaciones en PowerPoint (43\%), videos (36\%) y la clase magistral (23\%). Al no existir un estilo específico de aprendizaje en el aula se deberían utilizar estrategias o actividades que faciliten y complementen el aprendizaje, lo que permitirá que los estudiantes procesen mejor y de diversas formas la información.

A pesar de las limitaciones por el número de participantes, determinar los estilos de aprendizaje de los estudiantes de Ciencia del Suelo permitirá hacer una mejor planificación y aplicación de las estrategias de aprendizaje en los próximos cursos; es necesario indicar que aunque la muestra tomada correspondió a más del $95 \%$ de los estudiantes matriculados en esos períodos, sería conveniente ampliar la investigación sobre este tema en los futuros semestres.

\section{Conclusiones}

Para considerar una formación integral individualizada y grupal es primordial conocer los estilos de aprendizaje, y con base en ello plantear estrategias que mejoren el rendimiento de los estudiantes. Los alumnos con estilos de aprendizaje múltiples tienden a obtener mejor puntaje que aquellos en los que predomina un estilo de aprendizaje.

Las estrategias empleadas que tuvieron más impacto fueron la combinación de teoría y práctica. Mostrar un ambiente real en donde los estudiantes apliquen lo aprendido en clase motiva y capta la atención de los alumnos; otro recurso útil fue el uso de videos -que hacen la clase más dinámica y permiten aprender una temática amplia en un tiempo reducido. No obstante, la diversidad de estrategias usadas durante la clase es esencial para mejorar el desempeño de los estudiantes, incluso de aquellos que aprenden de manera diferente y que podrían ser una minoría, por ello la importancia de extender las estrategias de enseñanza, ya que esto amplía el rango de aprendizaje en la construcción del conocimiento. Debido al gran desafío que significa la enseñanza para los estudiantes de la Ciencia del Suelo, se requiere de una mayor investigación para determinar los estilos y estrategias de aprendizaje; los hallazgos de esta investigación constituirían un inicio para futuras investigaciones en el campo de la enseñanza-aprendizaje de la Ciencia del Suelo.

\section{Referencias}

Acosta, S. F. y García, M. Ch. (2012). Estrategias de enseñanza utilizadas por los docentes de biología en las universidades públicas. Omnia, 18(2), 67-82. Recuperado de http://www.redalyc.org/pdf/737/73723402005.pdf

Alonso, C., Gallego, D. y Honey, P. (1995). Los estilos de aprendizaje: procedimientos de diagnóstico y mejora. España: Mensajeros.

Amador, J. y Görres, J. (2004). A problema-based learning approach to teaching introductory Soil Science. Journal of Natural Resources and Life Sciences Education, 33, 21-27. Recuperado de https://www.agronomy.org/files/inrlse/issues/2004/e03-19.pdf

Balks, M. R. y Zabowski, D. (2016). Celebrating soil: discovering soils and landscapes. Suiza: Springer.

Chiang, M. T., Díaz, C. y Arriagada, P. (2016). Estilos de enseñanza y aprendizaje: ¿cómo dialogan en la práctica? Revista de Estilos de Aprendizaje, 9(17), 2-24. Recuperado de

http://learningstyles.uvu.edu/index.php/jls/article/view/260/202 
Czepulaz, A., Bottacin, W., Hipólito, E., Baptista, D., Pontarolo, R. y Correr, C. (2016). Predominant learning styles among pharmacy students at the Federal University of Paraná, Brazil. Pharmacy Practice, 14(1), 1-8. doi:10.18549/PharmPract.2016.01.650

Diochon, A., Basiliko, N., Krzic, M., Yates, T.T., Olson, E., Masse, J., Amiro, B., Kumaragamage, D. (2017). Profiling undergraduate soil science education in Canada?: Status and projected trends. Canadien Journal of Soil Science, 97(2), 122-132. doi.org/10.1139/cjss-2016-0058

Dunn, R. y Dunn, K. (1989). Learning style inventory. EUA: Price Systems.

Esguerra, G. y Guerrero, P. (2010). Estilos de aprendizaje y rendimiento académico en estudiantes de Psicología. Revista Diversitas-Perspectivas en Psicología, 6(1), 97-109. Recuperado de http://www.redalyc.org/articulo.oa?id=67916261008

Fazarro, D. E., Pannkuk, T., Pavelock, D. y Hubbard, D. (2009). The effectiveness of instructional methods based on learning style preferences of agricultural students: a research tool for continuous improvement for Faculty in Career and Technical Education (CTE) Programs. Journal of Industrial Teacher Education, 45(3), 84-104. Recuperado de http://Files.eric.ed.gov/Fulltext/EJ865355.pdf

Ferreiro, R. (2014). Cómo ser mejor maestro, el método Elı. México: Trillas.

Ferreiro, R. y Espino, M. (2014). El abc del aprendizaje cooperativo, trabajo en equipo para aprender y enseñar. México: Trillas.

Field, D. J., Koppi, A. J., Jarrett, L. E., Abbott, L. K., Cattle, S. R., Grant, C. D., McBratney, A. B., Menzies, N. W. y Weatherley, A. J. (2011). Soil Science teaching principles. Geoderma, 167-168, 9-14.

doi:10.1016/j.geoderma.2011.09.017

Fox, G. A., Kuster, E. L. y Fox, A. K. (2017). The importance of scientific publishing: teaching an undergraduate how to swim the entire length of the pool. Journal of Contemporary Water Research $y$ Education, 160, 1-4. doi:10.1111/j.1936-704X.2017.03236.x

García, J., Sánchez, C., Jiménez, M. y Gutiérrez, M. (2012). Estilos de aprendizaje y estrategias de aprendizaje: un estudio en discentes de postgrado. Revista Estilos de Aprendizaje, 10(10), 1-17.

Recuperado de http://www2.uned.es/revistaestilosdeaprendizaje/numero 10/articulos/Articulo06.pdf

Gallego, D. (2013). Ya he diagnosticado el estilo de aprendizaje de mis alumnos y ahora ¿qué hago? Revista Estilos de Aprendizaje, 12(11), 1-15. Recuperado de

http://www2.uned.es/revistaestilosdeaprendizaje/numero 12/articulos/articulo 1.pdf

Gaona-Flores, V. A., Campos-Navarro, L. A., Alcalá, E. y Ovalle-Luna, O. (2014). Transformative learning between the student and the teacher? Concerning the learning styles of medical residents. Global Advanced Research Journal of Medicine and Medical Science, 3(12), 445-450. Recuperado de http://gari.org/garimms/12/2014/3/12/transformative-learning-between-the-student-and-the-teacherconcerning-the-learning-styles-of-medical-residents

Hartemink, A., Balks, M., Chen, Z-S., Drohan, P., Field, D., Krasilnikov, P., Lowe, D., Rabenhorst, M., van Ress, K., Schipper, L., Sonneveld, M. y Walter, C. (2014). The joy of teaching soil science. Geoderma, 217218, 1-9. doi:10.1016/j.geoderma.2013.10.016

Hartemink, A. E. (2015). On global soil science and regional solutions. Geoderma Regional, 5, 1-3. doi:10.1016/j.geodrs.2015.02.001.

Hawk, T. y Shah, A. (2007). Using learning style instruments to enhance student learning. Decision Sciences Journal of Innovative Education, 5(1). doi:10.1111/j.1540-4609.2007.00125.x 
Honey, P. y Mumford, A. (1986). Using our learning styles. Reino Unido: Peter Honey Publications.

Hussain, N. y Ayub, N. (2012). Learning styles of students and teaching styles of teachers in business education: A case study of Pakistan. Procedia-Social and Behavioral Sciences, 69, 1737-1740.

doi.org/10.1016/j.sbspro.2012.12.122

International Union of Soil Sciences.(2016). 2015-2024 International decade of soils. Recuperado de http://iuss.boku.ac.at/files/iuss-bulletin128 72dpi.pdf

Joy, S. y Kolb, D. (2009). Are there cultural differences in learning style? International Journal of Intercultural Relations, 33(1), 69-85. doi.org/10.1016/j.ijintrel.2008.11.002

Lesmes-Anel, J., Robinson, G. y Moody, S. (2001). Learning preferences and learning styles: a study of Wessex general practice registrars. British Dental Journal, 51(468), 559-564. Recuperado de https://bjgp.org/content/51/468/559

Maldonado, S. (2014). Differences between Latino Students' Learning Styles and their gender. Institute for Learning Styles Journal, 1, 29-41. Recuperado de

https://www.auburn.edu/academic/education/ilsrj/Journal\%20Volumes/Fall\%202014\%20Vol\%201\%20P DFs/Learning\%20Styles\%20and\%20Gender\%20Maldonado\%20Torres.pdf

Mol, G. y Keesstra, S. (2012). Soil science in a changing world. Environmental Sustainability, 4(5), 473-477. Recuperado de https://www.sciencedirect.com/science/article/pii/S1877343512001509?via\%3Dihub

Mumford, A. y Honey, P. (1992). Questions and answers on learning styles questionnaire. Industrial and Commercial Training, 24(7), 10-13. Recuperado de

https://www.emeraldinsight.com/doi/pdfplus/10.1108/00197859210015426

Organización de las Naciones Unidas para la Alimentación y la Agricultura. (2015). Carta mundial de los suelos revisada. Recuperado de http://www.fao.org/3/b-i4965s.pdf

Pashler, H., McDaniel, D., Rohrer, D. y Bjork, R. (2009). Learning styles concepts and evidence. Psychological Science in the Public Interest, 9(3), 105-118. Recuperado de

https://www.psychologicalscience.org/journals/pspi/PSPI 9 3.pdf

Quichimbo, P., Chérrez, M. y Jiménez, L. (2016). Students's learning styles in soil science: an ecuadorian case study. Ecuador es Calidad, 4(Especial), 45-50. Recuperado de

http://www.agrocalidad.gob.ec/revistaecuadorescalidad/index.php/revista/article/view/47/86

Villalba, A. (2015). Estilos de aprendizaje en alumnos universitarios de profesorado en biología y licenciatura en biodiversidad. Revista de Estilos de Aprendizaje, 8(16), 79-100. Recuperado de http://learningstyles.uvu.edu/index.php/ils/article/view/241/194

Wade, W., Rasmussen, K. y Fox-turnbull, W. (2013). Can technology be a transformative force in education? Technology in Education, 57(3), 162-170. doi.org/10.1080/1045988X.2013.795790

Wilkinson, T., Boohan, M. y Stevenson, M. (2014). Does learning style influence academic performance in different forms of assessment? Journal of Anatomy, 224, 304-308. doi.org/10.1111/joa.12126

Wrenn, J. y Wrenn, B. (2009). Enhancing learning by integrating theory and practice. International Journal of Teaching and Learning in Higher Education, 2, 258-265. Recuperado de

http://files.eric.ed.gov/fulltext/EJ899313.pdf 